\title{
Performance of Subaru adaptive optics system AO188
}

\author{
Yosuke Minowa $^{a}$, Yutaka Hayano ${ }^{a}$, Shin Oya ${ }^{a}$, Makoto Watanabe ${ }^{b}$, Masayuki Hattori ${ }^{a}$, Olivier \\ Guyon $^{a}$, Sebastian Egner ${ }^{a}$, Yoshihiko Saito ${ }^{a}$, Meguru Ito ${ }^{a}$, Hideki Takami ${ }^{a}$, Vincent Garrel ${ }^{a}$, \\ Stephen Colley ${ }^{a}$, Taras Golota ${ }^{a}$ and Masanori Iye ${ }^{c}$ \\ ${ }^{a}$ Subaru Telescope, NAOJ, 650 North Aohoku Place, Hilo, Hawaii 96720, USA; \\ ${ }^{b}$ Hokkaido University, Faculty of Science, Kita 10 Nishi 8, Kitaku, Sapporo, Hokkaido \\ 060-0810, Japan; \\ ${ }^{c}$ National Astronomical Observatory of Japan (NAOJ), 2-21-1 Osawa, Mitaka, Tokyo \\ 181-8588, Japan
}

\begin{abstract}
Subaru adaptive optics system (AO188) is an 188-elements curvature sensor adaptive optics system that is operated in both natural and laser guide star modes. AO188 was installed at Nasmyth platform of the Subaru telescope and it has been successfully operating in the natural guide star mode since October 2008. The performance of AO188 in the natural guide star mode has been well verified from on-sky data obtained with the infrared camera and spectrograph (IRCS). Under normal seeing condition, AO188 achieves K-band Strehl ratio between $60 \%$ and $70 \%$ using $R=9.0$ magnitude natural guide stars and it works well with faint guide stars down to $R=16.5$ magnitude. We measured the FWHM and Strehl ratio of stellar images in globular clusters and found that the isoplanatic angle is approximately 30 arcsec. In this paper, we describe an overview of the operation procedure for AO188, as well as its performance such as angular resolution, Strehl ration, and sensitivity gain for detecting faint objects.
\end{abstract}

Keywords: adaptive optics, telescope operation, image quality, strehl ratio

\section{INTRODUCTION}

Subaru AO188 ${ }^{1}$ is a curvature adaptive optics system which consists of a 188 element wavefront curvature sensor with photon counting Avalanche Photo Diode modules and a 188 elements bimorph deformable mirror. It is installed at the IR Nasmyth platform of Subaru telescope and operated in both natural guide star (NGS) and sodium laser guide star (LGS) modes. The detail specification of the AO188 is summarized in Table 1. The science camera mainly used with AO188 is IRCS (Infrared Camera and Spectrograph), ${ }^{2}$ which provides imaging and spectroscopy capabilities in near-infrared $(0.9-5.3 \mu \mathrm{m})$ wavelength. HiCIAO (High Contrast Instrument for the Subaru Next Generation Adaptive Optics) ${ }^{3}$ is also available with AO188 as a PI type instrument. HiCIAO is equipped with a coronagraph and offers the capability of high contrast imaging at $0.9-2.5 \mu \mathrm{m}$ wavelength. We are also planning to use Kyoto-3DII ${ }^{4}$ with AO188 as a PI-type instrument, which provides the capability of integral field spectroscopy at optical wavelength range $(0.65-0.95 \mu \mathrm{m})$. AO188 has successfully completed the first light observations with IRCS in late 2006. AO188 NGS mode has been offered for open use observations since 2008B. Currently, LGS mode is in commissioning phase.

\section{AO188 OPERATION}

The operation of AO188 in NGS mode requires a guide star for the wavefront measurement. The guide star should be a bright star located within 30 arcsec from your target. The target object for the science camera can also be used as the guide star. To obtain reasonable performance improvement, the guide star should be brighter than $R$ magnitude of 16.5. Since we are using high-sensitive APDs for the wavefront sensor, the brightest limit of the guide star is around $R \sim 8$, although the brighter star up to $R \sim-1$ can be used as the guide star by

Further author information: (Send correspondence to Y.M.)

Y.M.: E-mail: minoways@subaru.naoj.org, Telephone: +1-808-934-5905

Adaptive Optics Systems II, edited by Brent L. Ellerbroek, Michael Hart, Norbert Hubin, Peter L. Wizinowich, Proc. of SPIE Vol. $7736,77363 N$ - @ 2010 SPIE - CCC code: 0277-786X/10/\$18 - doi: 10.1117/12.857818 
Table 1. Summary of AO188 NGS mode specifications

\begin{tabular}{|l|l|}
\hline Location of the system & Nasmyth focus (IR) \\
\hline Defomable mirror & Bimorph mirror with 188 electrodes \\
\hline Wavefront sensor & Curvature sensor with 188 photon counting APDs \\
\hline Real time computer & 4 Xeon CPUs $(2.0 \mathrm{GHz}$ ) with real time Linux OS \\
\hline Control band width & $>100 \mathrm{~Hz}, 1000$ correction/sec \\
\hline Field of view & $2^{\prime} .7$ \\
\hline Main optics throughput & $\sim 70 \%$ \\
\hline Guide star magnitude & $-1<R<16.5$ (NGS) \\
\hline Science instrument & IRCS, HiCIAO, Kyoto-3DII \\
\hline
\end{tabular}

using attenuation filters installed inside of the wavefront sensor. To feed the guide star to the wavefront sensor, we used a guide star acquisition unit that consists of two gimbal mirrors and a linear stage. The gimbal mirrors adjust the position and ray tilt of the guide star. The linear stage is mounted under the one of the gimbal mirrors and adjusts the focus position of the guide star by changing the separation of two gimbal mirrors. ${ }^{5}$

Once we acquire the guide star to the wavefront sensor, we try to find the optimum control parameters, namely amplitude of the focus modulation for wavefront curvature measurement and servo loop gain. The modulation amplitude is corresponding to the voltage applied to a vibrating mirror (VM) mounted in the wavefront sensor. Higher voltage enables the measurement of higher order wavefront aberrations, but decreases the $\mathrm{S} / \mathrm{N}$ of the wavefront measurement because of the loss of photon counts detected on the APDs. To find the optimum value, we scan the VM voltage by checking the photon counts. We empirically found that the optimum value is the voltage where the photon counts decreased by 10-20\% of the maxim um counts. Since AO188 is currently using the classical zonal control algorithm, we scan a single servo gain by checking wavefront error estimated at the wavefront sensor and the variance of the DM voltages. Figure 1 shows an example of the gain scan. Too low gain gives worse wavefront correction (i.e. large wavefront error), while too high servo gain gives control loop. We found that the DM variance and the wavefront error are good tracers for finding optimum gain that gives the highest Strehl ratio.

Typical overheads for acquiring the guide star and tuning the loop performance are 10 minutes for bright star $(R<14)$ and 15 minutes for faint stars $(R>14)$. If the guide star is an extended source (e.g., nucleus of AGN), additional $\sim 5$ minutes is required for the parameter tuning.

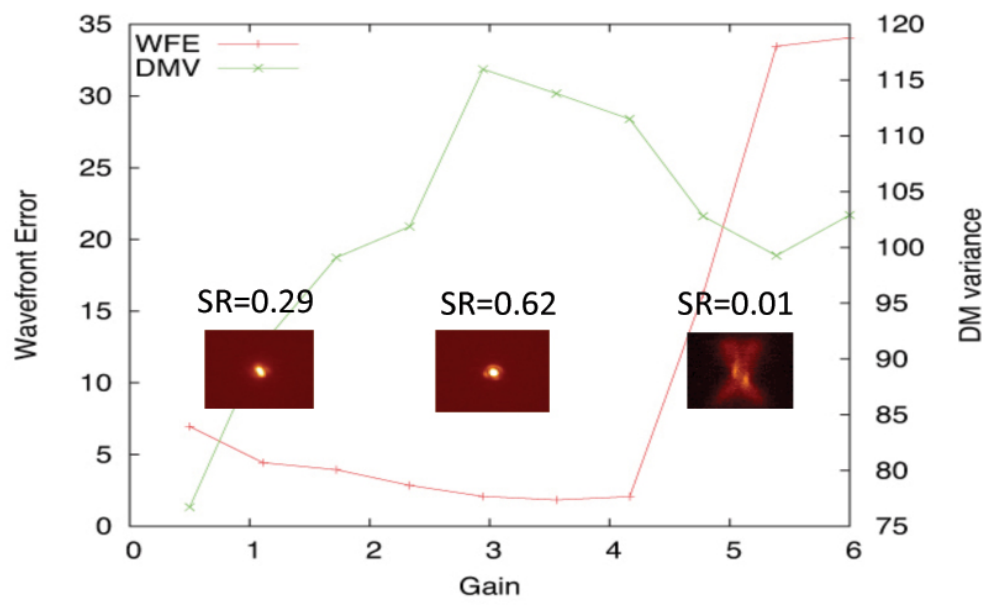

Figure 1. Example of the servo gain scanning. The images of PSF obtained with IRCS during the scanning are also shown. The optimization is performed by finding the gain at which the wavefront error is close to the smallest value before the DM control becomes unstable due to the oscillation. In this case, the gain of 3 gives the best performance. 


\section{PERFORMANCE EVALUATION}

AO188 was extensively tested during the engineering run held from 2008 to 2009 . The performance was evaluated in the near-infrared bands (zJHKL' $\mathrm{M}^{\prime}$ ) using IRCS image mode with a pixel scale of 20 mas/pix. We took point sources (stars) with various $R$-band magnitude from 8 to 17 . All exposures were longer than 10 seconds to get statistically meaningful data. To characterize AO188, we used the Strehl ratio and FWHM extracted from the IRCS images.

\subsection{Strehl ratio calculator}

The Strehl ratio is often used to characterize the error of AO system. It is defined as the ratio between the peak intensity of a PSF divided by the peak intensity of a diffraction-limited PSF with the same total flux. To calculate the Strel ratio, we need to find the peak value of the observed and ideal PSFs normalized by their total flux. However, it is difficult to find the peak value of the high Strehl ratio ( $>0.5)$ PSFs, because the pixel scale (20 mas/pix) of the IRCS is not small enough to well-sample the diffraction-limited PSFs whose FWHM is smaller than 60 mas. Moreover, it is difficult to find the total flux from the observed images from short $(\sim 10$ seconds) exposure images. The flux from the outer part of the PSF halo cannot be measured due to the noise and thereby underestimating the total flux (or overestimating the Strehl ratio). To overcome these difficulties, we have developed a code for measuring the Strehl ratio. At first we fits the observed PSF to find its peak value and center coordinates and measure the flux using a circular or elliptical aperture. The size and shape of the aperture are determined depending on the $\mathrm{S} / \mathrm{N}$ of the observed image using SExtractor software. ${ }^{6}$ Then a new image which only contains the ideal diffraction-limited PSF at the same coordinates with the same pixel scale (20 mas/pix) as the observed one are created. The ideal PSF is created by the fourier transform of the telescope pupil. A linear phase is applied on the fourier transfrom to shift the PSF. Figure 2 shows the example of the PSF pupil, the linear phase, and the calculated PSF. We measure the peak value from this "under-sampled" ideal PSF using the same fitting procedure for the observed PSFs. In order to take into account the effect of the total flux underestimation, we use the same aperture as the observed PSF for measuring the flux of the ideal PSF. Finally, we can get the Strehl ratio by dividing the peak to flux ratio of the observed PSF by that of the ideal PSF with less affected by the PSF under sampling and the flux underestimation.
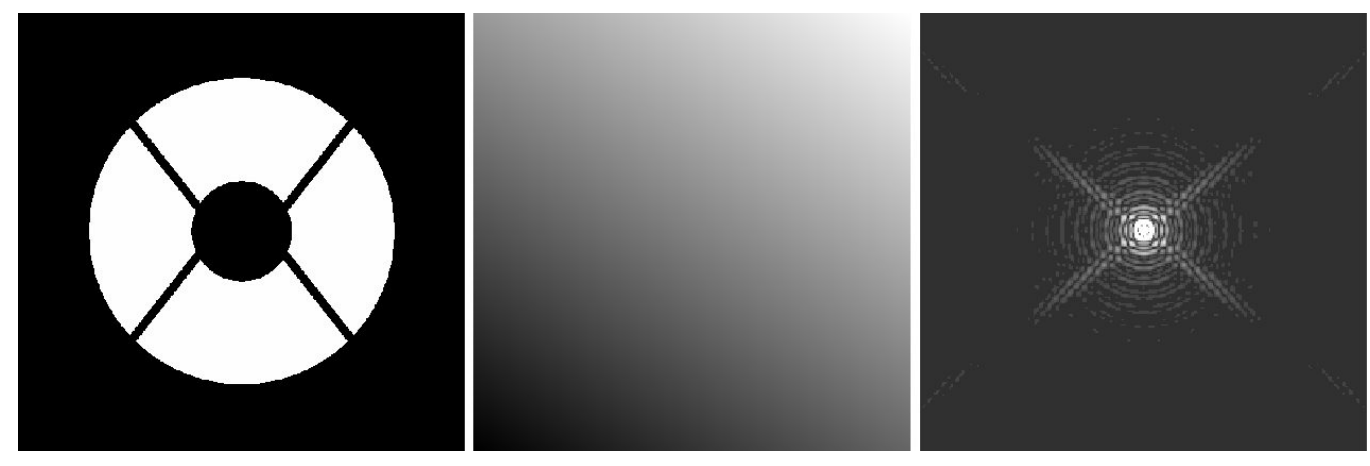

Figure 2. Examples of the telescope pupil (left) and linear phase (middle). The ideal PSF (left) was created by the fourier transform of the pupil and phase images.

\subsection{NGS on-source Strehl ratio and FWHM}

Figure 3 shows the achieved Strehl ratio and FWHM as a function of the R-band magnitude of the natural guide star and the observed wavelength. The Strehl ratio that we obtained for a bright guide star $(R<10 \mathrm{mag})$ was $1.0,0.85,0.65,0.40,0.20$ and 0.08 at $\mathrm{M}^{\prime}(4.86 \mu \mathrm{m}), \mathrm{L}^{\prime}(3.77 \mu \mathrm{m}), \mathrm{K}(2.20 \mu \mathrm{m}), \mathrm{H}(1.63 \mu \mathrm{m}), \mathrm{J}(1.25 \mu \mathrm{m})$, and $\mathrm{z}$ $(1.03 \mu \mathrm{m})$-bands, respectively, under seeing conditions of $0.4-0.7$ arcsec. We achieved the Strehl ratio of $\sim 0.1$ at $K$-band even for $R \sim 16.5$ faint guide star. The FWHM for all near-infrared bands were less than 0.1 arcsec except for the faintest guide star with $R \sim 16.5$, which is very close to the diffraction limit. Note that the FWHM of the shorter wavelength images might be overestimated because of the insufficient pixel sampling (20 mas/pix) with respect to the size of diffraction-limited images. 

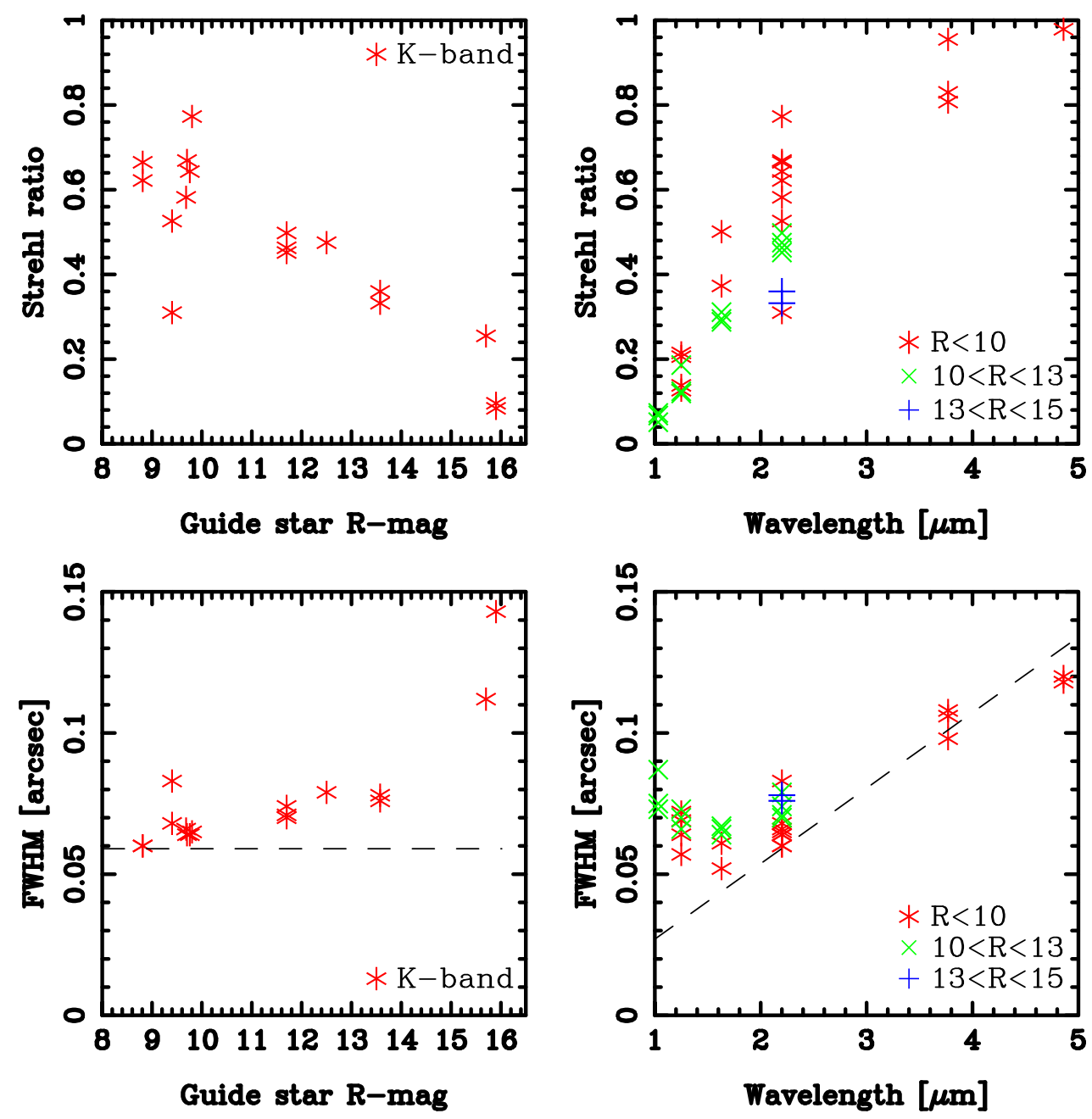

Figure 3. Strehl ratio as a function of $R$-band magnitude of the NGS and the observed wavelength under normal seeing condition of $0.4-0.7$ arcsec. Dashed lines show the FWHM of the ideal diffraction-limited (SR=1.0) PSF.

\subsection{Fried length versus Strehl ratio}

Figure 4 shows a plot of the Strehl ratio versus Fried length $\left(r_{0}\right)$ at the image wavelength. Solid lines show the model plot of the residual wavefront variance $(\Delta)$. The residual wavefront variance after removal of the $N$ th lower degrees of Zernike modes from Kormogorov phase screen is given by $\Delta(N)=0.2944 N-\sqrt{3} / 2\left(D / r_{0}\right)^{5 / 3}$ $\left[\mathrm{rad}^{2}\right]{ }^{7}$ We found that the best fit models are $\Delta /\left(D / r_{0}\right)^{5 / 3}=0.01$ to 0.02 for bright stars $(R<10)$ and 0.08 for faint stars $(R>15)$. These are corresponding to the correction up to the Zernike order of $20-50$ for bright stars and 5-6 for faint targets. Although we achieve the Strehl ratio of more than 0.7 in best case at $K$-band, our best performance is still low for the curvature sensor with 188 elements. According to the Racine $2006,{ }^{8}$ the curvature sensor with more than 100 sub-apertures is expected to achieve $\Delta /\left(D / r_{0}\right)^{5 / 3}<0.01$. This discrepancy could be cause by the non-common path error due to the difference in optical path between science and WFS paths and/or the limitation of the control bandwidth due to unexpected turbulence.

\subsection{Encircled energy and sensitivity gain}

Figure 5 shows the plot of the encircled flux as a function of the aperture radius in $z^{\prime} J H K$-bands with a typical $R=12$ th magnitude guide star. We found that the aperture radii that contains $50 \%$ of total flux are 4,3 , and 2 times smaller than typical seeing PSF (FWHM 0.4) at $K H, J$, and $z^{\prime}$-bands, respectively. These mean that the 


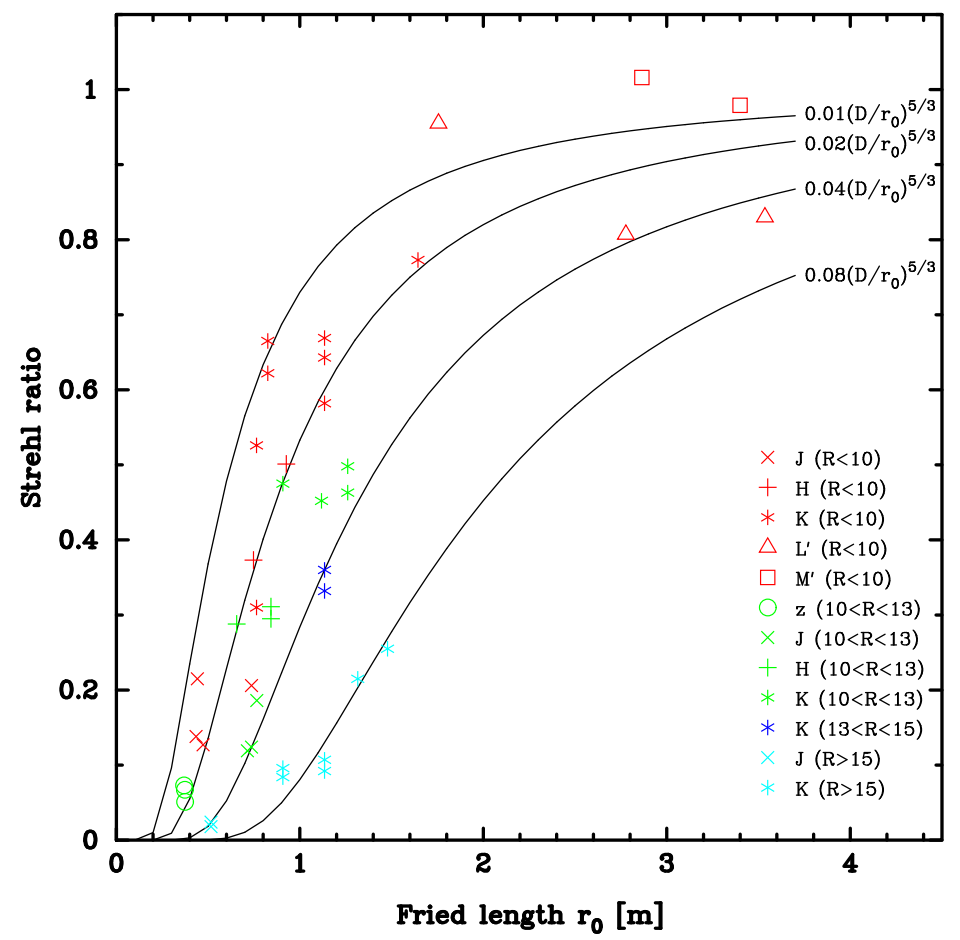

Figure 4. Strehl ratio versus Fried length $\left(r_{0}\right)$ at the near-infrared wavebands. Solid lines show the model predictions with the residual wavefront variances of $0.01,0.02,0.04$ and 0.08 .

sensitivity gain for detecting faint objects are increase by $0.75 \mathrm{mag}, 0.60 \mathrm{mag}$, and $0.37 \mathrm{mag}$ at $K H, J, z^{\prime}$-bands, respectively.

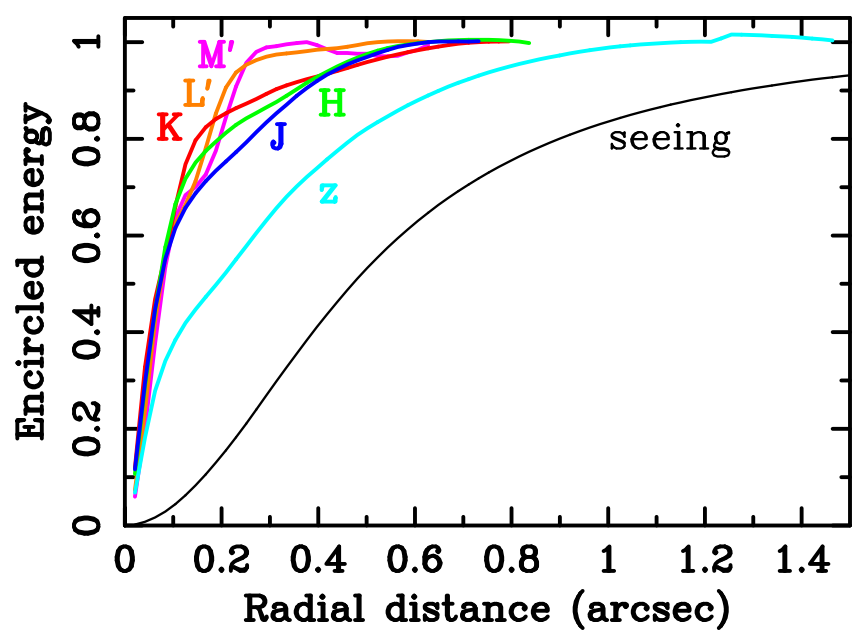

Figure 5. Encircled energy of the PSF as a function of the distance from the center of the AO corrected PSF at each near-infrared band. The encircled flux of a seeing PSF (FWHM 0'.4 arcsec) is also plotted for comparison. Total flux was normalized to unity.

\subsection{Isoplanatic Field}

Performance of AO correction degrades gradually with increasing distance from a guide star because the angular correlation of atmospheric turbulence becomes lower as increasing distance from a guide star (anisoplanatism). 
Figure 6 shows the Strehl ratio as a function of the distance from the guide star estimated from the image of globular cluster M5 and M15 obtained with IRCS 52 mas/pix mode. The Strehl ratio decreases with increasing the distance from the guide star $(\Delta \theta)$ by $S R \propto \exp \left[-\left(\Delta \theta / \theta_{0}\right)^{5 / 3}\right]$ where $\theta_{0}$ is an isoplanatic angle defined as the separation angle at which the Strehl ratio has degraded by a factor of e with respect to the on-axis value. We found that the isoplanatic angle of AO188 was around 30 arcsec in radius.

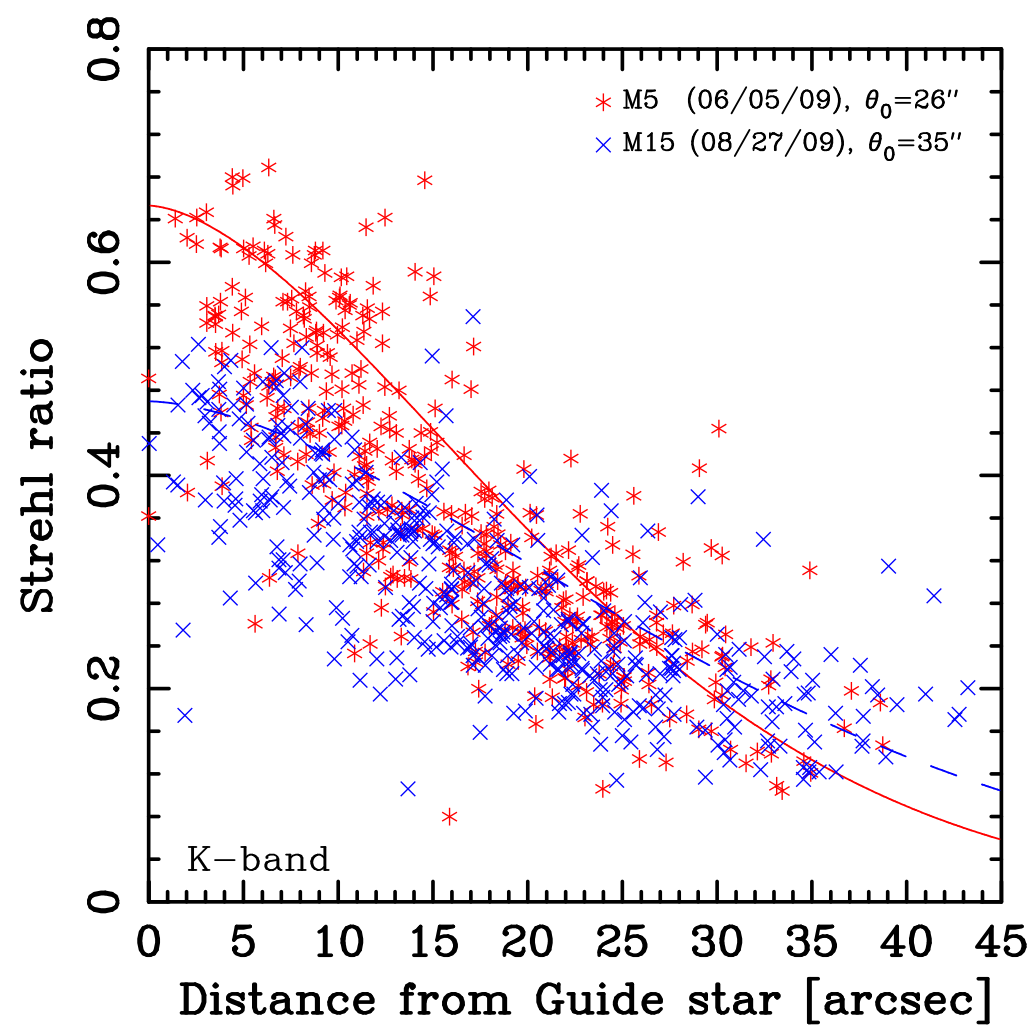

Figure 6. Variation of the Strehl ratio within IRCS 52mas/pix mode field of view as a function of distance from a guide star. The results are based on snapshots of globular clusters M15 (crosses) and M5 (asterisks) taken under normal seeing condition. The solid and dashed lines show the Strel ratio derived from the model of the residual wavefront variances as a function of the distance from guide stars with the isoplanatic angle of 26 arcsec and 35 arcsec, respectively.

\section{SUMMARY}

In this paper, we presented the procedure for AO188 operation such as the guide star acquisition and loop performance tuning. We also described a method for calculating the Strehl ratio which takes into account the effect of uncertainties in finding the peak and flux values from under-sampled short exposure images. Finally, AO188 NGS mode has been characterized from the on-sky performance such as FWHM and Strehl ratio of the PSF extracted from the IRCS near-infrared images.

\section{ACKNOWLEDGMENTS}

We would like to thank all staff of Subaru telescope, National Astronomical Observatory of Japan (NAOJ), who kindly support our research and development.

\section{REFERENCES}

[1] Hayano, Y., "Progress of the laser guide star adaptive optics at subaru telescope," in [Exopnalents and disks: their formation and diversity], T. Usuda, M. Tamura, . M. I., ed., American Institute of Physics Conference Series 1158, 385-386 (2009). 
[2] Kobayashi, N., Tokunaga, A. T., Terada, H., Goto, M., Weber, M., Potter, R., Onaka, P. M., Ching, G. K., Young, T. T., Fletcher, K., Neil, D., Robertson, L., Cook, D., Imanishi, M., and Warren, D. W., "Ircs: infrared camera and spectrograph for the subaru telescope," in [Optical and IR Telescope Instrumentation and Detectors], Iye, M. and Moorwood, A. F., eds., Proc. SPIE 4008, 1056-1066 (2000).

[3] Hodapp, K. W., Suzuki, R., Tamura, M., Abe, L., Suto, H., Kandori, R., Morino, J., Nishimura, T., Takami, H., Guyon, O., Jacobson, S., Stahlberger, V., Yamada, H., Shelton, R., Hashimoto, J., Tavrov, A., Nishikawa, J., Ukita, N., Izumiura, H., Hayashi, M., Nakajima, T., Yamada, T., and Usuda, T., "Hiciao: the subaru telescope's new high-contrast coronographic imager for adaptive optics," in [Ground-based and Airborne Instrumentation for Astronomy II], McLean, I. S. and Casali, M. M., eds., Proc. SPIE 7014, 42 (2008).

[4] Sugai, H., Hattori, T., Kawai, A., Ozaki, S., Hayashi, T., Ishigaki, T., Ishii, M., Ohtani, H., Shimono, A., Okita, Y., Matsubayashi, K., Kosugi, G., Sasaki, M., and Takeyama, N., "The kyoto tridimensional spectrograph ii on subaru and the university of hawaii 88 in telescopes," PASP 122, 103-118 (2010).

[5] Watanabe, M., Takami, H., Takato, N., Colley, S., Eldred, M., Kane, T., Guyon, O., Hattori, M., Goto, M., Iye, M., Hayano, Y., Kamata, Y., Arimoto, N., Kobayashi, N., and Minowa, Y., "Design of the subaru laser guide star adaptive optics module," in [Advancements in Adaptive Optics.], Domenico B. Calia, B. L. E. and Ragazzoni, R., eds., Proc. SPIE 5490, 1096-1104 (2004).

[6] Bertin, E. and Arnouts, S., "Sextractor: Software for source extraction.," A\&AS 117, 393-404 (1996).

[7] Noll, R. J., "Zernike polynomials and atmospheric turbulence," Journal of the Optical Society of America 66, 207-211 (1976).

[8] Racine, R., "The strehl efficiency of adaptive optics systems," PASP 118, 1066-1075 (2006). 